\title{
Molecular Epidemiology, Risk Factors and Outcomes of Carbapenem-Resistant Klebsiella Pneumoniae Infection in Eastern China: for A Retrospective Study Over 4 Years
}

\section{Dongmei Zhao}

First Affiliated Hospital of Anhui Medical University

\section{Hongru Li}

Xiangya Hospital Central South University

\section{Chengcheng Yue}

First Affiliated Hospital of Anhui Medical University

\section{Yanyan Liu}

First Affiliated Hospital of Anhui Medical University

Ying Ye

First Affiliated Hospital of Anhui Medical University

Jiabin Li ( $\square$ lijiabin@ahmu.edu.cn )

the first affiliated hospital of anhui medical university https://orcid.org/0000-0003-3238-569X

\section{Research article}

Keywords: Carbapenem-resistant Klebsiella pneumoniae, Epidemiology, Sequence type 11, Risk factors, Outcomes

Posted Date: September 8th, 2020

DOI: https://doi.org/10.21203/rs.3.rs-71433/v1

License: (c) (i) This work is licensed under a Creative Commons Attribution 4.0 International License. Read Full License 


\section{Abstract}

Objectives: Carbapenem-resistant Klebsiella pneumoniae (CRKP) have undergone extensive dissemination in worldwide resulting in increased mortality. We performed a retrospective analysis of epidemiology and risk factors for CRKP infection in a general teaching hospital in China.

Methods: A molecular and clinical study were conducted for 98 CRKP in a tertiary hospital from January 2013 to December 2016. Carbapenemase gene detection, pulsed-field gel electrophoresis (PFGE) and multilocus sequence typing (MLST) were performed. Logistic regression was also used to identify the risk factors associating with the 30-day mortality.

Results: The producition of KPC cabapenemase was the main resistant mechanism and increased annually with a significant difference. However, the molecular outcome revealed the dominance and diversity in CRKP with 24 sequence types (STs) and 59 PFGE types (PTs). The ST11 CRKP were documented as the predominant strains in our study, which showed a significant increasing trend year by year. Additionally, the predominant ST11 CRKP corresponding to PT10 and PT15 remained a typical fashion. Of note, the new advantage PT09 or PT16 were just discovered in 2016 which also corresponding to ST11. Meanwhile, the factors on 30-day mortality and ST11 proportionality with CRKP infection were assessed, which showed that ST11, appropriate empirical treatment, and hospital stays were independently associated with 30-day mortality.

Conclusions: ST11 CRKP payed a dominant role in the process, but the homology of these strains was polymorphic and the advantage clusters would be changed by evolution. Additionally, besides appropriate empirical treatment and hospital stays, ST11 CRKP was independently associated with the 30-day mortality, first reported as we know.

\section{Introduction}

Carbapenem-resistant Klebsiella pneumoniae (CRKP) has emerged as a major nosocomial pathogen and are being increasing reported worldwide [1-3], which has been associated with a high mortality rate range from approximately $16-70 \%[4,5]$. Carbapenem resistance in $K$. pneumoniae is reported to be caused either by epidemic clones or by the horizontal dissemination of mobile elements. Additionally, the predominant production of Klebsiella pneumoniae carbapenemase (KPC) contributed to the most important mechanism of carbapenem resistance in K. pneumoniae [6]. Of epidemiological significance, the international spread of KPC-producing $K$. pneumoniae is primarily associated with a single multilocus sequence type (ST) or its related variants. In Europe and America, KPC-producing ST258 K. pneumoniae is regarded as one of the most successful multidrug-resistant nosocomial pathogens [3]. However, the international high-risk clone of $K$. pneumoniae ST11 is frequently reported as a successful pathogen at infections in Asia [6-8]. Of note, Wang et al [9] reported that KPC-positive ST11 K. pneumoniae with a highly resistant to meropenem had a significant difference on the mortality. 
Indeed, data regarding the distribution of these resistant determinants among CRKP isolates collected during a longtime period is still limited in China, and the relationship between molecular characteristics and the clinical outcome is also sparing. In our study, we aimed to describe the epidemiology of CRKP, focus on the molecular characteristics of circulating strains and to evaluate which proportion of strains was susceptible to the clinical outcome.

\section{Methods}

\section{Study population}

A retrospective study for patients infected or colonized with CRKP from January 2013 to December 2016 was conducted in a hospital with 2800 beds. Medical charts were reviewed and demographic and clinical information was collected. This study was approved by the Ethics Committee of the first affiliated hospital of Anhui Medical University.

\section{Bacterial isolates and genotypic investigation of resistance}

Non-duplicate K. pneumoniae isolates which developed $48 \mathrm{~h}$ following hospital admission were collected. These isolates were subsequently characterized as hospital-acquired infections with resistant to imipenem, meropenem or ertapenem. Identification and antimicrobial drug susceptibility testing were performed using automated systems from VITEK®2 (bioMérieux, France). Organisms were considered resistant to carbapenems according to the CLSI guidelines (2016) [10]. Polymerase chain reaction (PCR) for the common $b / a_{K P C}$ gene was carried out in order to identify the carbapemenase gene of all CRKP.

\section{Molecular typing}

To track and characterize the clonal relationships of the K.pneumoniae isolates, pulsed-field gel electrophoresis (PFGE) of the Xbal-digested genomic DNA was conducted using a CHEF-DRIII system (Bio-Rad, United Kingdom). Strain relatedness analysis was performed on the BioNumerics software version based on the Dice coefficient for genetic relatedness and the unweighted-pair group method analysis using average linkages (UPGMA) for generation of dendrograms, which is described as the reference [11]. Concomitantly, multi locus sequence typing (MLST) was the most common technique, which based on genetic variation in seven housekeeping genes (rpoB, gapA, $m d h$, pgi, phoE, infB and ton $B$ ) that together provided a relative genetic profile. Then, CRKP isolates were assigned a ST number according to the allelic profiles available in the Institute Pasteur database. These ST data can be further defined by eBURST.

\section{Clinical characterization and definitions}

Clinical data was collected using electronic medical records which extract patient information, including demographic characteristics, chronic underlying diseases, invasive procedures, disease status, antimicrobial therapy, clinical outcomes with 30-day mortality. 1). Infection: all patients were evaluated 
according to CDC criteria [12] to assess whether the infection was due to CRKP. 2). Emergency state: the patient enters a hospital with the state of emergency disease, point to that the situation is urgent, illness is heavy, patient state is more dangerous. 3). Empirical and definitive therapy: treatment administered before the susceptibility testing results were available was characterized as empirical, whereas treatment given after the results were available (at least one active drug for $\geq 48 \mathrm{~h}$ ) was considered as definitive therapy. The former is divided into appropriate treatment and inappropriate treatment in our study. Appropriate antibiotic treatment was defined as treatment with at least one agent for $\geq 48 \mathrm{~h}$ after the isolation of a clinical culture specimen to which the isolate was susceptible to in vitro [13]. Definitive treatment regimens were classified as monotherapy (treatment with one in vitro active agent) and combination therapy (treatment with two or more in vitro active agents), and the latter included associated with Tigecycline or with others. 4). Clinical outcome: Infection-related mortality was defined as the proportion of patients who died only as a direct consequence of the CRKP infection, and with no other plausible explanation in the opinion of the local investigator. Therefore, 30-day infection-related mortality was considered as death for patients who died within 30 days of the onset of the CRKP infection.

\section{Statistical analysis}

Statistical analysis was performed using SPSS version 21 software (IBM Corp., Armonk, NY). Univariate analysis was performed to identify factors related to mortality and the ST11 of CRKP. The chi-square test was used for categorical variables and Student's t-test for continuous variables. A logistic regression model was used to identify factors independently associated with mortality. Sets of variables that had $P$ $\leq 0.1$ in the univariate analysis on mortality were entered into the model. P-values were interpreted together with $95 \%$ confidence interval $(\mathrm{Cl})$ for the logistic regression model. All tests were two tailed and a P-value of $<0.05$ was considered statistically significant.

\section{Results}

\section{Isolates}

A total of 98 patients with CRKP infection were identified during the study period. Of these, the median age was 62 years, with nearly three-quarters were male, and 42 patients $(42.9 \%)$ were admission to intensive care unit (ICU). The 98 non-duplicate CRKP isolates were obtained from sputum samples (72.4\%), blood cultures (13.3\%) and other samples (14.3\%). Based on antibiotic resistance testing for these isolates, imipenem MICs ranged from $2 \mathrm{mg} / \mathrm{mL}$ to $16 \mathrm{mg} / \mathrm{mL}$ (two intermediate isolates), or meropenem MICs ranged from $4 \mathrm{mg} / \mathrm{mL}$ to $16 \mathrm{mg} / \mathrm{mL}$, or ertapenem MICs present $2 \mathrm{mg} / \mathrm{mL}$. Antimicrobial alternatives to carbapenems demonstrating through automated systems included the resistant rates of $100 \%$ to ampicillin/sulbactam and ceftriaxone, $98.0 \%$ to ceftazidime, more than $93 \%$ to ceftazidime, aztreonam, ciprofloxacin and piperacillin/tazobactam. Amikacin, gentamicin and levofloxacin showed $80.6 \%, 82.7 \%$ and $88.78 \%$, respectively. And all isolates except two intermediate ones were susceptible to Tigecycline. Of these isolates, 60 (61.2\%) yielded a clinical specimen with the 
predominant $b / a_{K P C}$ positive CRKP and 55 isolates (56.1\%) were classified into ST11. During the three periods across the year of 2013 to 2016, we found that CRKP infection had no statistical significance in the distribution of age, gender, ICU admission and sample source, however, the CRKP isolates carrying $b / a_{K P C}$ showed an increasing trend year by year, and which showed significantly different between the three periods $(p<0.001)$. Accordingly, a significantly higher prevalence of isolates containing ST11 CRKP $(p<0.001)$ was also noted in the near year (Table 1$)$.

\section{Characterization of CRKP isolates by Molecular analysis}

In this study, 24 different STs were identified in these $98 \mathrm{~K}$. pneumoniae isolates by MLST analysis, the most dominant sequence type was ST11 (56.1\%, 55/98), followed by ST23 $(11.2 \%, 11 / 98)$, ST15 and ST86 (3.1\%, 3/98 respectively). And each of the 6 STs belonged to 2 isolates, while 14 STs obtained 1 strain, respectively. Among these, 10 STs of strains produced KPC $\beta$-lactamase, of which the predominant ST11 harbored 49 KPC-producing K. pneumoniae isolates $(89.1 \%, 49 / 55)$ and ST23 contained 4 KPCpositive ones $(36.4 \%, 4 / 11)$, the remaining STs possessed only one, respectively.

Of the 98 isolates, 3 isolates were ruled out for the degradation of chromosome nucleic acid. Thus, 95 were available for PFGE, and the analysis revealed 59 different clonal PFGE types (PTs, PT01-PT59). Of those, the highest percentage clone was named PT15 (15.8\%, 15/95), followed by PT10 (8.4\%, 8/95), PT06 and PT11 (4.2\%, 4/95, respectively), PT13 (3.2\%, 3/95), and PT01, PT04, PT07, PT08, PT09, PT16 and PT36 (2.1\%, 2/95, respectively), the remaining PTs possessed only one, respectively. Interestingly, ST11 also occupied predominant isolates according to the prepotent PFGE pattern. 54 CRKP isolates (the actual was 55 strains for one nucleic acid degradation) of 23 PTs clones corresponded to the same type of ST11, the top type clones were recommended as PT15 (25.9\%, 14/54), PT10 (13.0\%, 7/54), PT06 and PT11 (7.4\%, 4/54, respectively). According to clinical departments, total of 16 STs were observed in ICU admission, the ST11 $(57.1 \%, 24 / 42)$ and ST23 $(7.1 \%, 3 / 42)$ commanded the foremost position, harboring 25 (59.5\%, 25/42) KPC-2-positive K. pneumoniae isolates. As well, the dominant clone PT10 and PT15 corresponded to the advantage type of ST11 in ICU. It is worthy noting that the only two strains of CRKP with clone type of PT09 or PT16 were just discovered from ICU admission in 2016 which corresponding to ST11 (Figure 1).

\section{Outcome and ST11}

During the study period, 87 patients with CRKP infection were included in the analysis of clinical outcome, while the remaining ones were excluded due to incomplete electronic information or for the inability to follow up with the study. With available outcome data, 32 patients $(36.8 \%, 32 / 87)$ died within 30 days of the onset of the CRKP infection, while 55 patients $(63.2 \%, 55 / 87)$ did not die within the time. To identify potential risk factors for 30-day mortality, infected patients who died due to CRKP infection were compared with the survived patients (Table 2$)$. The 30 -day mortality was mainly male $(71.9 \%$, $23 / 87$ ) with a mean \pm standard deviation age $60.19 \pm 21.31$ years. On univariate analysis, no significant difference in sex, age, specimen source, underlying disease, emergency state, invasive operation, 
combination with other strain and definitive therapy were observed. However, the length of hospitalization was associated with 30 -day mortality with a certain significance $(p=0.088)$, as well as the admission to ICU $(p=0.061)$. Of note, the 30-day mortality of patients treated with appropriate empirical treatment was $9.4 \%(3 / 32)$ and the survivor was $45.5 \%(25 / 55)$, which showed a significant difference $(p<0.001)$.

Due to the tendency of ST11 CRKP in hospital mentioned above, we analyzed the possible risk factors depending on clinical data. ST11 CRKP and non-ST11 CRKP were compared in Table 2. The patients with ST11 CRKP infection had a significant emergency state than those with non-ST11 $(p=0.03)$. Additionally, the operative treatment and appropriate empirical treatment both showed a borderline significance with the ST11 CRKP infection ( $p=0.075$ or $p=0.056$, respectively). Then, no significant statistical difference in other related variables was found between the ST11 CRKP and non-ST11 strains.

A logistic regression analysis was conducted in order to investigate the association between the 30-day mortality and risk factors, including the length of hospitalization, ICU admission, appropriate empirical treatment, emergency state and ST11 CRKP. The results revealed that the length of hospitalization (odds ratio [OR], 0.961; 95\% confidence interval [CI] 0.935-0.988; $P=0.004)$, appropriate empirical treatment (OR, $11.301 ; 95 \% \mathrm{Cl}, 2.47-51.71 ; P=0.002)$ and ST11 CRKP (OR, 0.193; 95\% Cl, 0.056-0.669; $P=0.01$ ) were factors independently associated with 30-day mortality. Admission to ICU and emergency state were not found with significant statistical difference (Table 3).

\section{Discussion}

Nosocomial infections due to CRKP are associated with substantial mortality, yet the clinical significance of isolating CRKP from the hospital is unknown. To our knowledge, this study is the first to describe the epidemiology and 30-day mortality of patients with CRKP infection from the long-term retrospective observation. Through this extensive inquiry, a clear picture has emerged which gradually reveal the particularly noteworthy findings. First of all, due to the trend of the KPC-positive CRKP strains belonging to the dominant ST11 lineage were observed, thus, our investigation took further insight on the epidemiology. The outcome stressed the diversity in CRKP strains with 24 clones of ST and 59 clusters of PT, however, the strains of predominant ST11 CRKP corresponding to PT10 and PT15 remained in vogue. Importantly, it is worthy noting that the new advantage cluster of PT09 or PT16 were just discovered from ICU admission in 2016 which corresponding to ST11. Secondly, The 30-day mortality of the patients due to CRKP infection was associated with the pathogens of ST11 CRKP. Summary together, our findings provided a new insight into the risk factors of the clinical outcome and further demonstrated the importance of epidemiology.

In our study, the investigation was conducted on CRKP isolates in 2013-2016. The molecular epidemiological methods had been used for tracking and characterization of CRKP isolates by PFGE and MLST which were accepted to differentiate the species-both of which indicated that CRKP ST11 was identified as the predominant sequence type strain in our study. The epidemiology of CRKP is continually evolving, and now the advantage strains of bla $a_{K P C}$-producing $K$. pneumoniaeST11 have been reported, 
which is the most frequently detected KPC-producing K. pneumoniae clone in China [14-16]. Interestingly, ST11 also occupied predominant isolates according to the prepotent PFGE pattern. As well, the dominant clone PT10 and PT15 corresponded to the advantage type of ST11 in ICU where took a important role in acquisition of carbapenem resistant isolates [17]. It is worthy noting that the only two strains of CRKP with clone type of PT09 or PT16, only second to the dominant cluster PT, were just discovered from ICU admission in 2016 which corresponding to ST11. In this study, it stated that ST11 CRKP payed a dominant role in the process, but aslo emphasized that the homology of these strains was polymorphic and the advantage clusters were changed by evolution. Naturally, an efficient measure could be established to monitor the variation of the prevalence of these CRKP strains.

In this report, the overall 30-day mortality rate of patients infected with CRKP was $36.78 \%$ (32/87), which was similar to that reported by previous studies for CRKP infections $[18,19]$. Several previous studies [20-22] had showed that the long-term hospitalization and empirical treatment were independent predictors of death. Through our retrospective study, the length of hospitalization and appropriate empirical treatment both were independently associated with 30-day mortality. However, we would not provide further evidence for this. The key point of this study was to evaluate the ST11 CRKP. It was unexpectedly that the mortality was with respect to the pathogens of ST11 CRKP, which was first reported, as we know so far.

Next, this focused investigation on the ST11 CRKP would be taken into consideration. Sequence types of globally prevalent carbapenem-resistant Enterobacteriaceae, including ST11 of K. pneumoniae clonal complex 258, were considered as a high-risk clones [23]. Thus, we believe that the analysis of type ST11 and the association with clinical baseline factor were concerning. Though the underlying mechanisms behind the emergence of CRKP ST11 clones, as well as their associations with the outcomes are yet to be elucidated, we used our acquisition of knowledge by reviewing the relevant literature and attempted to provide some perspectives on the issue. Closely related to ST258, ST11's factors that contribute to the epidemiologic success remain unknown. However, several articles report that chromosomal or plasmid factors, beyond antibiotic resistance, may increase the strain's fitness and provide an advantage that underlies its prevalence [24, 25]. Additionally, a recent report [26] showed that the KPC-producing $K$. pneumoniae ST11 clone was resistant to serum killing, which may also be proof for the prevalence. In addition, a review [27] explained that ST11 genome strains carried distinct capsular polysaccharide (cps) regions, and the cps locus was one of the primary determinants of antigenicity associated with $K$. pneumoniae. Capsule switching was a species-specific mechanism used by the microbe to escape the host immune response. Croucher, et al [28] inferred that DNA exchange in-and-around the cps regions may be an important mechanism used by K. pneumoniae to rapidly diversify and evolve. Therefore, the success prevalence of CRKP ST11 may be due largely to chromosomal recombination instead of antibiotic resistance. Brisse $S$, et al [29] found that strains owning some serotypes usually carried a relevant virulence factor content and had been associated with serious human infections, which underscored the potential threat represented by the emergence and diffusion of carbapenem-resistance clones with increased virulence potential [30,31]. Undoubtedly, careful monitoring of carbapenem 
susceptibilities and rapid identification of epidemiological lineages was quite necessary for implementation of infection-control measures to prevent the endemicity of CRKP.

Our study has several limitations. This paper does not unify the the patients of CRKP infection, difference from the other observational studies, which were based on available data that include only patients with bacteremia or bacteriuria [32-34]. Additionally, for the fewer strains acquired from the saved isolates in 2014, the study appeared to be incomplete. And, our further research should take insight into the virulent CRKP ST11 strains. Together this research will allow us to expand our knowledge on such strains.

In conclusion, ST11 CRKP payed a dominant role in the process, but the homology of these strains was polymorphic and the advantage clusters would be changed by evolution. Additionally, besides appropriate empirical treatment and hospital stays, ST11 CRKP was independently associated with the 30-day mortality. Therefore, efforts are imminently needed to improve the understanding to the knowledge of the epidemiological status to prevent its spread.

\section{Abbreviations}

CRKP, Carbapenem-resistant Klebsiella pneumoniae, PFGE, pulsed-field gel electrophoresis; MLST, multilocus sequence typing; KPC, Klebsiella pneumoniae carbapenemase; ST, sequence type; PT, PFGE type; CLSI, Clinical and Laboratory Standards Institute; PCR, Polymerase chain reaction; ICU, intensive care unit.

\section{Declarations}

\section{Authors' contributions}

Prof. Jiabin Li contributed substance to ideas and design; Dongmei Zhao and Hongru Li contributed equally to this work, drafted the paper or responsible for the experiment; Chengcheng Yue and Yanyan Liu collected some clinical material; Ying Ye offered help in test operation.

\section{Acknowledgements}

Not applicable.

\section{Competing interests}

The authors declare that they have no competing interests.

\section{Availability of data and materials}

Not applicable.

\section{Consent for publication}


All authors have seen and approved for publication.

\section{Ethics approval and consent to participate}

This study was approved by the Ethics Committee of the first affiliated hospital of Anhui Medical University (Quick- PJ 2017-11-19) and consent to participate.

\section{Funding}

This work was supported by the National Natural Science Foundation of China (Grant No.81673242)

\section{References}

1. Logan LK, Weinstein RA. The epidemiology of carbapenem-resistant Enterobacteriaceae: the impact and evolution of a global menace. J Infect Dis. 2017;215:28-36.

2. Tängdén T, Giske CG. Global dissemination of extensively drug-resistant carbapenemase-producing Enterobacteriaceae: clinical perspectives on detection, treatment and infection control. J Intern Med. 2015;277:501-12.

3. Tzouvelekis LS, Markogiannakis A, Psichogiou M, Tassios PT, Daikos GL. Carbapenemases in Klebsiella pneumoniae and other Enterobacteriaceae: an evolving crisis of global dimensions. Clin Microbiol Rev. 2012;25:682-707.

4. Ben-David D, Kordevani R, Keller N, Tal I, Marzel A, Gal-Mor O, et al. Outcome of carbapenem resistant Klebsiella pneumoniae bloodstream infections. Clin Microbiol Infect. 2012;18:54-60.

5. Petrosillo N, Giannella M, Lewis R, Viale P. Treatment of carbapenem-resistant Klebsiella pneumoniae: the state of the art. Expert Rev Anti-Infect Ther. 2013;11:159-77.

6. Munoz-Price LS, Poirel L, Bonomo RA, Schwaber MJ, Daikos GL, Cormican M, et al. Clinical epidemiology of the global expansion of Klebsiella pneumoniae carbapenemases. The Lancet infectious diseases. 2013;13:785-96.

7. Qi Y, Wei Z, Ji S, Du X, Shen P, Yu Y. ST11, the dominant clone of KPC-producing Klebsiella pneumoniae in China. J Antimicrob Chemother. 2010;66:307-12.

8. Andrade LN, Curiao T, Ferreira JC, Longo JM, Clímaco EC, Martinez R, et al. Dissemination of blaKPC2 by the spread of Klebsiella pneumoniae clonal complex 258 clones (ST258, ST11, ST437) and plasmids (IncFII, IncN, IncL/M) among Enterobacteriaceae species in Brazil. Antimicrob Agents Chemother. 2011;55:3579-83.

9. Wang X, Wang Q, Cao B, Sun S, Zhang Y, Gu B, et al. Retrospective Observational Study from a Chinese Network of the Impact of Combination Therapy versus Monotherapy on Mortality from Carbapenem-Resistant Enterobacteriaceae Bacteremia. Antimicrob Agents Chemother. 2018;63. pii: e01511-e01518.

10. Wayne PA. Clinical and Laboratory Standards Institute Performance standards for antimicrobial susceptibility testing: twenty-sixth informational supplement M100-S26. CLSI, 2016. 
11. Han H, Zhou H, Li H, Gao Y, Lu Z, Hu K, et al. Optimization of pulse-field gel electrophoresis for subtyping of Klebsiella pneumoniae. Int J Environ Res Public Health. 2013;10:2720-31.

12. Horan TC, Andrus M, Dudeck MA. CDC/NHSN surveillance definition of health care-associated infection and criteria for specific types of infections in the acute care setting. Am J Infect Control. 2008;36:309-32.

13. Zarkotou O, Pournaras S, Tselioti P, Dragoumanos V, Pitiriga V, Ranellou K, et al. Predictors of mortality in patients with bloodstream infections caused by KPC-producing Klebsiella pneumoniae and impact of appropriate antimicrobial treatment. Clin Microbiol Infect. 2011;17(12):1798-803.

14. Pitout JD, Nordmann P, Poirel L. Carbapenemase-producing Klebsiella pneumoniae: a key pathogen set for global nosocomial dominance. Antimicrob Agents Chemother. 2015;59:5873-84.

15. Liu J, Yu J, Chen F, Yu J, Simner P, Tamma P, Liu Y, Shen L. Emergence and establishment of KPC-2producing ST11 Klebsiella pneumoniae in a general hospital in Shanghai, China. Eur J Clin Microbiol Infect Dis. 2018;37:293-9.

16. Sun K, Chen X, Li C, Yu Z, Zhou Q, Yan Y. Clonal dissemination of multilocus sequence type 11 Klebsiella pneumoniae carbapenemase-producing K. pneumoniae in a Chinese teaching hospital. Apmis. 2015;123:123-7.

17. Katchanov J, Asar L, Klupp EM, Both A, Rothe C, König C, et al. Carbapenem-resistant Gram-negative pathogens in a German university medical center: prevalence, clinical implications and the role of novel $\beta$-lactam/ $\beta$-lactamase inhibitor combinations. PLoS ONE. 2018;13:e0195757.

18. Giannella M, Trecarichi EM, Giacobbe DR, De Rosa FG, Bassetti M, Bartoloni A, et al. Effect of combination therapy containing a high-dose carbapenem on mortalityin patients with carbapenemresistant Klebsiella pneumoniae bloodstream infection. Int J Antimicrob Agents. 2018;51:244-8.

19. Chiu S-K, Chan M-C, Huang L-Y, Lin Y-T, Lin J-C, Lu P-L, et al. Tigecycline resistance among carbapenem-resistant Klebsiella Pneumoniae: Clinical characteristics and expression levels of efflux pump genes. PloS one. 2017;12:e0175140.

20. Tumbarello M, Viale P, Viscoli C, Trecarichi EM, Tumietto F, Marchese A, et al. Predictors of mortality in bloodstream infections caused by Klebsiella pneumoniae carbapenemase-producing $\mathrm{K}$. pneumoniae: importance of combination therapy. Clin Infect Dis. 2012;55(7):943-50.

21. Tumbarello M, Trecarichi EM, De Rosa FG, Giannella M, Giacobbe DR, Bassetti M, et al. Infections caused by KPC-producing Klebsiella pneumoniae: differences in therapy and mortality in a multicentre study. J Antimicrob Chemother. 2015;70:2133-43.

22. Girometti N, Lewis RE, Giannella M, Ambretti S, Bartoletti M, Tedeschi S, et al. Klebsiella pneumoniae bloodstream infection: epidemiology and impact of inappropriate empirical therapy. Medicine. 2014;93:298-309.

23. Mathers AJ, Peirano G, Pitout JD. The role of epidemic resistance plasmids and international highrisk clones in the spread of multidrug-resistant Enterobacteriaceae. Clin Microbiol Rev. 2015;28:56591. 
24. Chmelnitsky I, Shklyar M, Hermesh O, Navon-Venezia S, Edgar R, Carmeli Y. Unique genes identified in the epidemic extremely drug-resistant KPC-producing Klebsiella pneumoniae sequence type 258. Journal of antimicrobial chemotherapy. 2012;68:74-83.

25. Cottell JL, Saw HT, Webber MA, Piddock LJ. Functional genomics to identify the factors contributing to successful persistence and global spread of an antibiotic resistance plasmid. BMC microbiology. 2014;14:168.

26. Chiang T-T, Yang Y-S, Yeh K-M, Chiu S-K, Wang N-C, Lin T-Y, et al. Quantification and comparison of virulence and characteristics of different variants of carbapenemase-producing Klebsiella pneumoniae clinical isolates from Taiwan and the United States. Journal of Microbiology Immunology Infection. 2016;49:83-90.

27. Chen L, Mathema B, Chavda KD, DeLeo FR, Bonomo RA, Kreiswirth BN. Carbapenemase-producing Klebsiella pneumoniae: molecular and genetic decoding. Trends in microbiology. 2014;22:686-96.

28. Croucher NJ, Klugman KP. The emergence of bacterial "hopeful monsters". MBio. 2014;5(4):e0155014.

29. Brisse S, Fevre C, Passet V, Issenhuth-Jeanjean S, Tournebize R, Diancourt L, et al. Virulent clones of Klebsiella pneumoniae: identification and evolutionary scenario based on genomic and phenotypic characterization. PloS one. 2009;4:e4982.

30. Zhang Y, Zeng J, Liu W, Zhao F, Hu Z, Zhao C, et al. Emergence of a hypervirulent carbapenemresistant Klebsiella pneumoniae isolate from clinical infections in China. J Infect. 2015;71:553-60.

31. Zhang R, Lin D, Chan EW-C, Gu D, Chen G-X, Chen S. Emergence of carbapenem-resistant serotype K1 hypervirulent Klebsiella pneumoniae strains in China. Antimicrob Agents Chemother. 2016;60:70911.

32. Tofas P, Skiada A, Angelopoulou M, Sipsas N, Pavlopoulou I, Tsaousi S, et al. Carbapenemaseproducing Klebsiella pneumoniae bloodstream infections in neutropenic patients with haematological malignancies or aplastic anaemia: Analysis of 50 cases. Int J Antimicrob Agents. 2016;47:335-59.

33. Daikos GL, Tsaousi S, Tzouvelekis LS, Anyfantis I, Psichogiou M, Argyropoulou A, et al. Carbapenemase-producing Klebsiella pneumoniae bloodstream infections: lowering mortality by antibiotic combination schemes and the role of carbapenems. Antimicrob Agents Chemother. 2014;58:2322-8.

34. Qureshi ZA, Syed A, Clarke LG, Doi Y, Shields RK. Epidemiology and clinical outcomes of patients with carbapenem-resistant Klebsiella pneumoniae bacteriuria. Antimicrob Agents Chemother. 2014;58:3100-4.

\section{Tables}


Table 1

Carbapenem-resistant Klebsiella pneumoniae case characteristics.

\begin{tabular}{|c|c|c|c|c|c|}
\hline & Total & 2013-2014 & 2015 & 2016 & \multirow[t]{2}{*}{$P$ value } \\
\hline & $(n=98, \%)$ & $(n=24, \%)$ & $(n=36, \%)$ & $(n=38, \%)$ & \\
\hline $\begin{array}{l}\text { Age (years) } \\
\text { (mean } \pm \text { S.D.) }\end{array}$ & $61.58 \pm 19.07$ & $61.46 \pm 22.16$ & $63.49 \pm 17.09$ & $58.90 \pm 19.06$ & 0.728 \\
\hline Gender (male) & $72(73.5)$ & $18(75)$ & $27(75)$ & $27(71.1)$ & 0.911 \\
\hline ICU of admission & $42(42.9)$ & $10(41.7)$ & $16(44.4)$ & $16(42.1)$ & 0.971 \\
\hline Specimen source & & & & & 0.945 \\
\hline Sputum & $71(72.4)$ & $17(70.8)$ & $26(72.2)$ & $28(73.7)$ & \\
\hline Blood & $13(13.3)$ & $3(12.5)$ & $4(11.1)$ & $6(15.8)$ & \\
\hline Others & $14(14.3)$ & $4(16.7)$ & $6(16.7)$ & $4(10.5)$ & \\
\hline KPC-2 & $60(61.2)$ & $4(16.7)$ & $23(63.9)$ & $33(86.8)$ & $<0.001$ \\
\hline ST11 & $55(56.1)$ & $4(16.7)$ & $22(61.1)$ & $29(76.3)$ & $<0.001$ \\
\hline
\end{tabular}


Table 2

Univariate analysis of factors associated with 30-day mortality and ST11 for 87 patients with carbapenemase-resistant Klebsiella pneumoniae infection.

\begin{tabular}{|c|c|c|c|c|c|c|}
\hline & $\begin{array}{l}\text { 30-day- } \\
\text { mortality } \\
(n=32, \%)\end{array}$ & $\begin{array}{l}30 \text { - day- } \\
\text { survivor } \\
(n=55, \%)\end{array}$ & $\begin{array}{l}P \\
\text { value }\end{array}$ & $\begin{array}{l}\text { ST11 } \\
(n=53, \\
\%)\end{array}$ & $\begin{array}{l}\text { Non- } \\
\text { ST11 } \\
(n=34, \\
\%)\end{array}$ & $\begin{array}{l}P \\
\text { value }\end{array}$ \\
\hline Gender, male & $23(71.9)$ & $42(76.4)$ & 0.642 & $\begin{array}{l}38 \\
(71.7)\end{array}$ & $\begin{array}{l}27 \\
(79.4)\end{array}$ & 0.75 \\
\hline $\begin{array}{l}\text { Age (years) } \\
\text { (mean } \pm \text { S.D.) }\end{array}$ & $\begin{array}{l}60.19 \pm \\
21.31\end{array}$ & $\begin{array}{l}63.73 \pm \\
15.60\end{array}$ & 0.376 & $\begin{array}{l}60.19 \pm \\
19.22\end{array}$ & $\begin{array}{l}65.91 \pm \\
15.16\end{array}$ & 0.146 \\
\hline $\begin{array}{l}\text { The length of hospitalization } \\
\text { (mean } \pm \text { S.D.) }\end{array}$ & $\begin{array}{l}32.5 \pm \\
21.69\end{array}$ & $\begin{array}{l}40.02 \pm \\
29.35\end{array}$ & 0.088 & $\begin{array}{l}37.87 \pm \\
24.30\end{array}$ & $\begin{array}{l}39.53 \pm \\
31.24\end{array}$ & 0.782 \\
\hline ICU admission & $17(53.1)$ & $18(32.7)$ & 0.061 & $\begin{array}{l}24 \\
(45.3)\end{array}$ & $\begin{array}{l}11 \\
(32.4)\end{array}$ & 0.23 \\
\hline Specimen source & & & 0.421 & & & 0.261 \\
\hline Sputum & 22 & 41 & & 40 & 23 & \\
\hline Blood & 6 & 5 & & 8 & 3 & \\
\hline Others & 4 & 9 & & 5 & 8 & \\
\hline Underlying disease & $24(75)$ & $42(76.4)$ & 0.886 & $\begin{array}{l}39 \\
(73.6)\end{array}$ & $\begin{array}{l}27 \\
(79.4)\end{array}$ & 0.535 \\
\hline Emergency state & $23(71.9)$ & $35(63.6)$ & 0.432 & $\begin{array}{l}40 \\
(75.5)\end{array}$ & $\begin{array}{l}18 \\
(52.9)\end{array}$ & 0.03 \\
\hline Operative treatment & $10(31.3)$ & $14(25.5)$ & 0.56 & $\begin{array}{l}11 \\
(20.8)\end{array}$ & $\begin{array}{l}13 \\
(38.2)\end{array}$ & 0.075 \\
\hline Invasive operation & $5(15.6)$ & $15(27.27)$ & 0.213 & $\begin{array}{l}14 \\
(26.4)\end{array}$ & $6(17.7)$ & 0.343 \\
\hline $\begin{array}{l}\text { Combination with other } \\
\text { bacterium }\end{array}$ & $23(71.9)$ & $33(60)$ & 0.605 & $\begin{array}{l}35 \\
(66.0)\end{array}$ & $\begin{array}{l}21 \\
(61.8)\end{array}$ & 0.685 \\
\hline $\begin{array}{l}\text { Appropriate empirical } \\
\text { treatment }\end{array}$ & $3(9.4)$ & $25(45.45)$ & $\begin{array}{l}< \\
0.001\end{array}$ & $\begin{array}{l}13 \\
(25.4)\end{array}$ & $\begin{array}{l}15 \\
(44.1)\end{array}$ & 0.056 \\
\hline Definitive therapy & & & 0.521 & & & 0.121 \\
\hline Monotherapy & 6 & 15 & & 12 & 9 & \\
\hline Combination with Tigecycline & 19 & 26 & & 24 & 21 & \\
\hline $\begin{array}{l}\text { Combination with } \\
\text { carbapenem }\end{array}$ & 7 & 14 & & 17 & 4 & \\
\hline
\end{tabular}


Table 3

Factors associated with 30-day mortality in 87 patients with carbapenemase-resistant Klebsiella pneumoniae infection by Logistic regression model.

\begin{tabular}{|lllll|}
\hline Variables & Odds ratio & \multicolumn{2}{c|}{$95 \%$ Confidence interval } & \multirow{2}{*}{$\boldsymbol{P}$ value } \\
\cline { 3 - 4 } & & lower & upper & \\
\hline The length of hospitalization & 0.961 & 0.935 & 0.988 & 0.004 \\
\hline ICU admission & 0.381 & 0.095 & 1.52 & 0.171 \\
\hline Emergency state & 1.19 & 0.283 & 5.007 & 0.812 \\
\hline Appropriate empirical treatment & 11.301 & 2.47 & 51.71 & 0.002 \\
\hline ST11 & 0.193 & 0.056 & 0.669 & 0.01 \\
\hline
\end{tabular}

Figures 


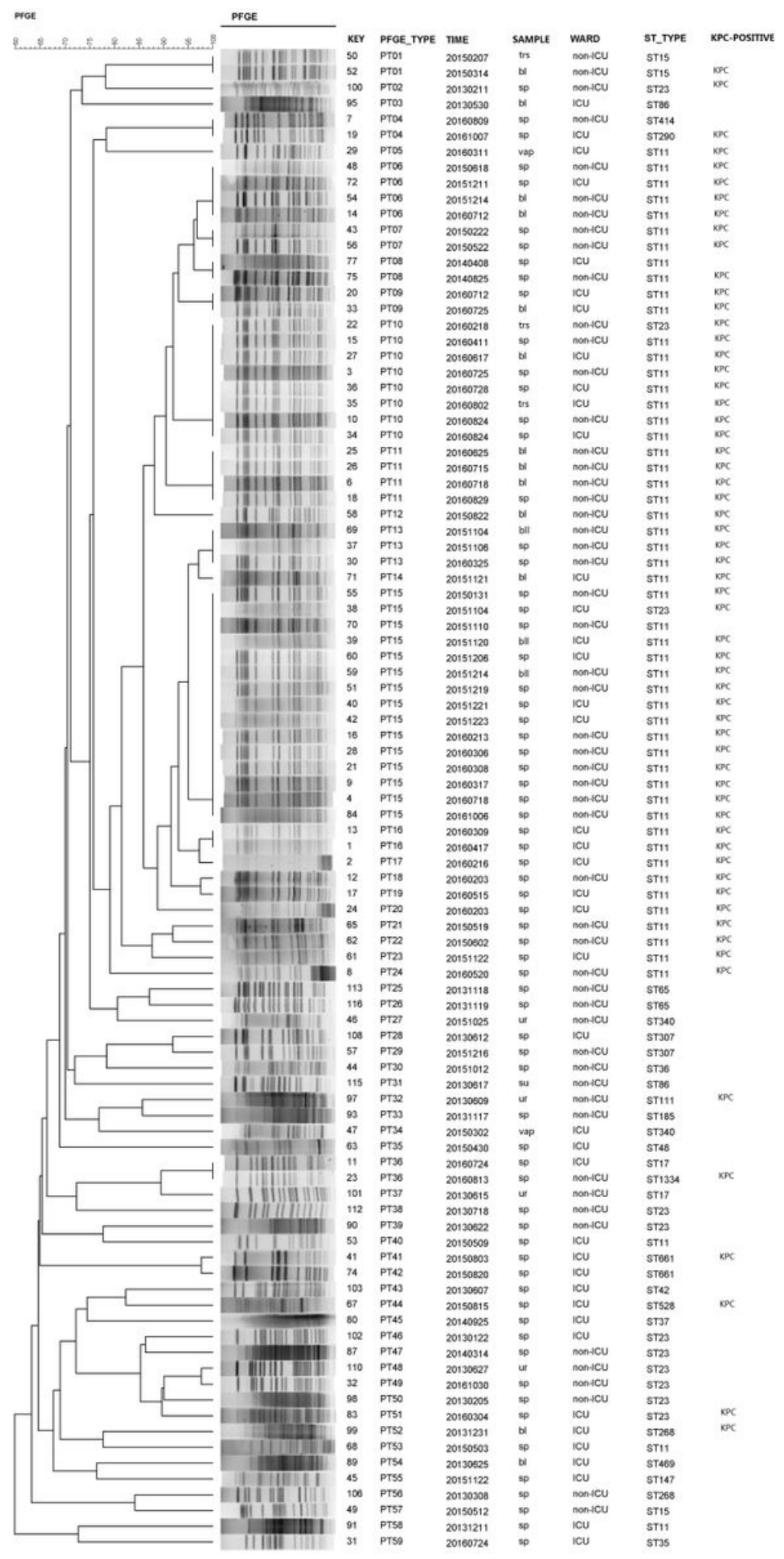

Figure 1

PFGE dendrogram generated with Bionumerics software showing the genetic relationship between 95 strains of carbapenem-resistant Klebsiella pneumoniae. The analysis of the bands generated was performed by using the Dice similarity coefficient and the unweighted pair group method with arithmetic averages. 